\title{
„Wenn mans zum ersten Mal hört, dann verchlüpft man automatisch."1 Eine Gratwanderung zwischen Sterbehelfer und Mörder. Essayistische Überlegungen zu Alices Reise in die Schweiz von Lukas Bärfuss
}

\author{
"When you hear it for the first time, you automatically get \\ frightened." A Tightrope Walk between Death Assistant and \\ Murderer. An Essay on Alice's trip to Switzerland by Lukas Bärfuss
}

Jan Trna

\begin{abstract}
This essay deals with the stage play "Alices Reise in die Schweiz" (Alice's trip to Switzerland) by Lukas Bärfuss, specifically focusing on the main character Gustav. There is a question raised whether he actually acts for purely ethical reasons, as he pretends, or whether something else is behind it. To formulate it more accurately: Is he as a suicide doctor tending to act like a murder? If so, where is the dividing line between these two positions? And how does it change the accustomed approach to the author Bärfuss who allegedly raises ethical issues without clearly taking sides?
\end{abstract}

\section{Keywords}

Lukas Bärfuss, Alices Reise in die Schweiz, ethical issues, euthanasia in Switzerland

Man kann Lukas Bärfuss nicht die Gabe absprechen, in der Öffentlichkeit stark resonierende Fragen in literarische Form gekonnt in literarische Form umzusetzen. So traf 2005

1 BÄRFUSS, Lukas: Alices Reise in die Schweiz. Die Probe. Amygdala. Stücke. Göttingen 2007, S. 9-57, hier S. 36 . 
auch sein Stück Alices Reise in die Schweiz den Nerv der Debatten um den assistierten Suizid. ${ }^{2}$ Den Blickwinkel verschärft Bärfuss in seinem Stück noch dadurch, dass die zentrale Rolle nicht etwa nur durch eine schwerstkranke Patientin mit auswegloser Diagnose besetzt ist, sondern eine junge depressive Frau aus Hamburg namens Alice ins Zentrum der Handlung gestellt wird. Als ihr Begleiter in den Tod agiert der Schweizer Arzt Gustav, der, nachdem er Alices Todeswunsch erfüllt hat, auch ihre fürsorgliche Mutter Lotte von dem Vor-sich-Hinvegetieren ,befreit', da sich eine weitere Existenz ohne ihre Tochter für sie erübrigt hat.

Dabei stellt sich die Frage, inwieweit Gustav im Stück tatsächlich als Hilfestellung leistende Person auftritt bzw. in welchem Maße seine Motivationen moralisch zu verurteilen sind. Um dies noch klarer auf den Punkt zu bringen, wird in diesem Essay versucht zu erkunden, wo sich die Trennlinie zwischen selbstlosem Handeln, als das Gustav seine Taten unentwegt darzustellen pflegt, und einem Handeln liegt, das durch diverse, ethisch fragwürdige Beweggründe kontaminiert wird. Die Klärung dieser Fragestellung kann Betrachtungsweisen offenlegen, die Bärfuss oft unterstellte Deutungsmuster untergraben, er stelle moralische Themen in den Raum, ohne sie eindeutig zu beantworten. ${ }^{3}$ Hätte sich Gustavs Neigung zum ,Mörderischen' bestätigt, hätte eine moralisch unparteiische, neutrale Deutung dieses Stücks ein für alle Mal festen Boden verloren.

\section{Gustav als unzulänglicher Beschützer der menschlichen Würde}

Die gängige Definition eines Einzelgängers mag auf Gustav gut zutreffen, denn er allein ${ }^{4}$ hatte neben seiner gewöhnlichen Arztpraxis ${ }^{5}$ in einem Wohnmobil den sterbewilligen Patienten eine tödliche Dosis Natrium Pentobarbital verabreicht, ${ }^{6}$ bevor ihm Walter, ein ausschließlich auf finanziellen Gewinn ausgerichteter Mann, eine Wohnung zur Verfügung stellt. Außer seiner Mitgliedschaft in der Ärztekammer fühlt sich Gustav weder

2 Vgl. SCHÄCHTER, Reinhard: Die Euthanasiedebatte in der Schweiz. In: BECKMANN, Rainer [et al] (Hg.): Sterben in Würde. Beiträge zur Debatte über Sterbehilfe, S. 259-272.

3 Vgl. GUNREBEN Marie; MARX Friedhelm: Handlungsmuster der Gegenwart. Eine Einleitung. In: GUNREBEN, Marie; MARX Friedhelm (Hg.): Handlungsmuster der Gegenwart. Beiträge zum Werk von Lukas Bärfuss. Würzburg 2017, S. 9.

4 Außerdem hat er noch eine gescheiterte Ehe hinter sich.

5 Lediglich an einer Stelle wird erwähnt, dass die Menschen sich nicht von einem Doktor Tod behandeln lassen wollen, was darauf schließen lässt, dass er eine auf die Heilung orientierte ärztliche Tätigkeit betreibt. Im Stück wird allerdings nur seine „Sterbehelferei“ fokussiert.

6 Die ist die einzige in der Schweiz erlaubte Weise, indem der sog. assistierter Suizid oder die Beihilfe zur Selbsttötung geleistet wird, d.h. obwohl das Tötungsmittel und alles mit dem Tod Zusammenhängende vom Sterbehelfer vorbereitet wird, muss der Patient den finalen Schritt selber tun - etwa den Hahn drehen, der ihm das Gift mittels einer Infusion in seinen Kreislauf appliziert. Dies geschieht allerdings bereits ohne körperliche Präsenz des Helfenden, der sich dem Todesort fernhalten muss, um nicht der Unterlassung einer Hilfestellung bezichtigt werden zu können. Vgl. BEDFORD-STROHM, Heinrich: Leben dürfen - leben müssen. Argumente gegen die Sterbehilfe. München 2015, S. 40 ff. 
an Institutionen ${ }^{7}$ noch an den öffentlichen Diskurs ${ }^{8}$ gebunden. Er sieht sich als einsamer Hüter der menschlichen Würde; stigmatisiert in den Augen der Öffentlichkeit und drangsaliert von den Ermittlungsbehörden. Wie oben angesprochen, lässt er sich durch keinerlei Zweifel beirren und klammert bereits im Vorfeld alle störenden Faktoren aus, indem er etwa seine Patienten davor warnt, Familienangehörige als Begleitung mitzubringen, die „unersprießliche Diskussion [...] über Sinn und Zweck und Statthaftigkeit“9 der Sterbehilfe anzetteln und dadurch den glimpflichen Vorgang des Aus-dem-LebenScheidens gefährden würden.

Gustavs legaler Betätigung wird ein Ende gesetzt, nachdem er sich der depressiven Alice annimmt. Dies hat den Entzug seiner Lizenz und Ausschluss aus der Ärztekammer $^{10}$ zur Folge, wodurch ihm der Zugang zu nötigen Arzneien verwehrt bleibt. Somit muss die tödliche Infusion mit Pentobarbital durch eine Plastiktüte abgelöst werden, die sich der Sterbewillige nach Einnahme von Analgetika aufsetzt und folglich erstickt. Um der Würde ${ }^{11}$ des Menschen willen verlässt Gustav die juristische Grauzone und gerät in den Bereich des Strafbaren. Dabei ist es paradoxerweise für sein Tun symptomatisch, dass er vor würdelosen Bedingungen nicht zurückschreckt - sei es das Wohnmobil, in dem er zuerst seine Arbeit verrichtet, oder später dann eine schäbige Wohnung in einem verruchten Viertel ${ }^{12}$. Weder den Räumlichkeiten noch seinem Äußeren wird große Bedeutung beigemessen. Ständig akzentuiert wird dagegen die Person des Sterbewilligen. Zudem legt die neue Tötungsmethode eindeutiges Zeugnis über die vielleicht nicht intendierte Missachtung der menschlichen Würde ab: Wer sich auf Gustav Sterbehilfeansatz einlässt, muss damit rechnen, mit einer über den Kopf gezogenen Plastiktüte am Boden liegend aufgefunden zu werden und demzufolge die Möglichkeit in Betracht ziehen, dass eine gerichtsärztliche Obduktion angeordnet wird. An dieser Stelle kommt, wie beim außer Acht gelassenen Umfeld, die ästhetische Seite des assistierten Suizids zum Vorschein. G. Vorjans bemerkt dazu, dass „[d]ie Suizidart in einem literarischen Text [...] auch die Funktion erfüllen [kann], eine bestimmte Ästhetik des Todes und der Darstellung der Leiche zu ermöglichen. " ${ }^{13}$ Der Grund, weshalb sich die potenziellen Suizidenten für solch einen Tod entscheiden, mag nämlich auch daran liegen, dass sie ihren Mitmenschen kein traumatisierendes Erlebnis beim Anblick ihres verunstalteten Leichnams bereiten wollen. Obwohl man vor diesen Konsequenzen in Gustavs Praxis

7 Dignitas oder Exit sind beispielsweise Namen der Organisationen, die in der Schweiz Sterbehilfe in ihrem Angebot führen.

8 „Wie sie vielleicht wissen, hat man mich in jüngster Zeit ins Licht der Öffentlichkeit gezerrt, ich wollte das nicht, die Medien können höchstens ein Mittel zum Zweck sein. "BÄRFUSS [Anm. 1], S. 10 f.

9 BÄRFUSS [Anm. 1], S. 10.

10 Die Begründung des Beschlusses der Ärztekammer besagt, er beeinträchtige durch sein Verhalten die durchaus nützliche Diskussion über die Sterbehilfe für psychisch Kranke. Vgl. BÄRFUSS [Anm. 1], S. 32.

11 Ein sehr oft in Bezug auf Sterbehilfe erwähnter Begriff, dessen lateinische Variante die Organisation Dignitas sogar in ihrem Namen führt.

12 Alice: „Ich kam an zwei Sexshops vorbei, und überall diese Abfallberge. Das ist ein schlechtes Viertel, dieses Viertel.“ Gustav: „Darauf habe ich dich vorbereitet.“ Bärfuss [Anm. 1], S. 48.

13 VORJANS, Gerrit: Von der Torheit, wählerisch zu sterben. Suizid in der deutschsprachigen Literatur um 1900. Bielefeld 2016, S. 336. 
geschützt bleibt, muss sich der Sterbewillige mit starken Einbußen in seiner Würde zurechtfinden.

\section{Gustav als geschickter Manipulator}

Die Position der Befürworter der Sterbehilfe erscheint viel leichter vertretbar, wenn man für den Fall einer stark physisch leidenden, kurz vor dem Tod stehenden Person plädiert. Ist nämlich aus klinischen Beobachtungen und ärztlicher Erfahrung unmissverständlich darauf zu schließen, dass das Leben des Betroffenen Aussichten auf eine Besserung entbehrt, mag ein durch einen ärztlichen Eingriff bewerkstelligtes Lebensende viel berechtigter erscheinen als der Suizid einer körperlich kerngesunden Person, die von psychischem Leiden geplagt wird. In erster Linie ist zu bemerken, dass einem solchen Menschen unvergleichbar mehr Zeit zur Verfügung steht, in der die Wahrscheinlichkeit durchaus vorhanden ist, dass eine Wende zur Besserung eintritt. Hinzugefügt werden muss auch die Tatsache, der das psychische Schmerz sich genauso wie der physische durch Medikation lindern lässt, wenn auch mögliche Schäden für die Seele in Kauf genommen werden. Immerhin handelt es sich bei Depressionen, die zur Lebensmüdigkeit führen, um einen langwierigen und schwer zu beeinflussenden Prozess. ${ }^{14}$ Auch für diese Fälle steht Gustav als Fürsorger und Fürsprecher und letztlich auch als Sterbehelfer resolut im Sinne der von T. Macho gestellten, offenen Frage ein:

Warum sind so viele Wörter mit dem Präfix ,für' aus unserem alltäglichen Sprachschatz verschwunden oder haben pejorative Bedeutungsverschiebungen erlitten. Warum sind ,Fürsorger' oder ,Fürsprecher' so unbeliebt? Werden sie vielleicht mit Vormundschaft, Paternalismus und Entmündigung verknüpft. ${ }^{15}$

Gustav entwickelt Strategien, die solchen Gefühlen entgegentreten sollten. So holt er seine Patienten nie vom Bahnhof ab, damit sie nicht dazu neigen, als Eingefangene in einem Prozess zu agieren und damit jederzeit ein Umdenken bei ihnen erfolgen könnte. Es soll eine Befreiung von allen äußeren Zwängen angestrebt werden, indem der zum Suizid Entschlossene eine vorbehaltlose Bewilligung von den nächsten Familienangehörigen erlangen muss. Somit stärkt Gustav seine vermeintlich neutrale Position, die auf der einen Seite allen Wünschen der Betroffenen entgegenkommen möchte, auf der anderen allen Kräften nach versucht, die Hinterbliebenen vor negativen Empfindungen zu schonen.

Anders gedeutet, es wird einem einfachen Muster gefolgt: Die Stellungnahme des Patienten wird vorbehaltlos angenommen, wobei die Meinung der Widersacher des Suizids aus den Reihen der Angehörigen entweder gar nicht zugelassen (siehe oben) oder zu Gunsten des assistierten Suizids hinterfragt wird. Als Gustav in Erfahrung bringt,

14 Vgl. MACHO, Thomas: Das Leben nehmen. Suizid in der Moderne. Berlin 2017, S. 10.

15 Ebd., S. 10. 
Alices Mutter Lotte sei mit der Entscheidung ihrer Tochter nicht einverstanden, zielt er bei seinem Überredungsversuch eben auf die Ästhetik des Sterbens ab:

Kommen Sie zur Vernunft. Eines Tages wird es Ihrer Tochter gelingen. Sie wird Ihnen entschlüpfen. Sie werden sich an jedem Tag vielleicht für ein Nickerchen hinlegen, oder kurz eine Besorgung machen, und plötzlich wird es sehr still sein. Sie werden nach Alice rufen, aber sie wird nicht antworten. Sie werden sie in der Badewanne finden, weiß im blutroten Wasser, auf dem Dachboden, erhängt, zerschmettert im Hof. Davor möchte ich sie bewahren. ${ }^{16}$

Mit unverhohlener Lust am Beschreiben versucht Gustav, Vorstellungen in Lotte heraufzubeschwören, die ihr wegen des Verhaltens von Alice mit hoher Wahrscheinlichkeit schon früher zu schaffen machten - Alice solle nämlich schon mehrmals einen apparativen, also absichtlich nicht vollzogenen Suizid begangen haben, um Aufmerksamkeit auf ihr erbärmliches Befinden zu lenken. Dies wird vom Sterbehelfer zunutze gemacht und durch eine bildhafte Beschreibung verdeutlicht. Kaum Abwendbares stehe an, er allein sei derjenige, der diese für alle Seiten schmerzhafte Lage behutsam und professionell auflösen könne. Statt Alices ,Hilferufe‘, die in ihrer suizidalen Verhaltensweise Abdruck fanden, als Anlass zu einer tieferen ärztlichen Auseinandersetzung ${ }^{17} \mathrm{zu}$ nehmen, dem Ziel nachstrebend, die junge Frau wieder ins Boot des Lebens zu holen, baut Gustav zu ihr ein Vertrauensverhältnis auf. Vielmehr kann und sollte man die absichtlich nicht erfolgreichen Selbstmordversuche in erster Linie als Wille zum Leben deuten. Wenn man auf die quasi sicheren, von Gustav beschriebenen, Tötungsmethoden verzichtet und sich stattdessen einer theatralen zuwendet, muss ein basales Hängen an der eigenen Existenz noch vorhanden sein und somit auch das Potential für eine Besserung des gesundheitlichen Zustands.

Deutet man dieses Erzwingen zur Einwilligung der Sterbeabsicht als einen mehr oder minder gelungenen Manipulationsversuch, so muss gleichzeitig auch ein anderer erwähnt werden. Bevor es nämlich zu der bereits geschilderten Begegnung kam , hatte sich Alice im Gespräch mit ihrer Mutter einer Lüge bedient, die anscheinend Gustavs Vertrauenswürdigkeit stärken sollte. Bei der ersten Erwähnung des Namens ihres Sterbehelfers geht es folgendermaßen zu:

ALICE Und deshalb hilft mir Gustav beim Sterben.

LOTTE Gustav. Bestimmt ein falscher Name.

ALICE Das ist sein Vorname. Strom ist sein Nachname.

LOTTE Ihr duzt euch.

ALICE Warum nicht.

16 BÄRFUSS [Anm. 1], S. 29.

17 Über Gustavs Approbation findet man im Text keine Auskunft, trotzdem liegt es auf der Hand, dass er diesbezüglich Meinungen seiner Kollegen aus dem Bereich der Psychologie hätte heranziehen können. Dies tut er jedoch nicht, zieht diese Option auch nicht in Erwägung. 
LOTTE Du duzt sonst keinen.

ALICE Er hat es mir angeboten. ${ }^{18}$

Durch den Sachverhalt, dass jemand Alices Zuneigung so schnell gewonnen hat, obwohl sie ständig alle männerkonnotierten Vorschläge im Vorfeld kompromisslos abschlägt, scheint es gerechtfertigt, darauf zu schließen, dass die Einstellung der Mutter zu Gustav damit beeinflusst werden sollte. Etwas später wendet sich Gustav an Lotte, ohne der deklarierten Lockerung der Beziehung gerecht zu werden: „Denken Sie an Lotte.“19

Gustavs besagtes, ohne weiteres zustimmendes Herangehen an Alices Entscheidung erweist sich umso kritikträchtiger, wenn ihre vollständige Abhängigkeit von ihrer Mutter Lotte mit bedacht wird. Ihre Repliken Alice („Du solltest diese Dinge nicht ohne mich in Angriff nehmen. Du lässt dich zu Dingen überreden, bist beeinflussbar. ${ }^{(20}$ ) oder Gustav gegenüber („Ich brauche sie nicht zu hindern. Sie wird freiwillig bei mir bleiben." $\left.{ }^{\text {"1 }}\right)$ legen unmissverständlich an den Tag, wie sicher sie sich ihres Einflusses auf die eigene Tochter ist. In diesem Lichte scheint es plausibel zu sein, die Äußerung von T. Macho heranzuziehen: „Ich mache das Leben zu meinem Leben, selbst indem ich es auslösche.“22 Der Kampf um Alices Leben wird augenscheinlich viel mehr von ihrer Mutter ausgetragen als von Alice selbst. Für sie ist dieses Ringen schon längst zu einer lästigen, sinnentleerten Angelegenheit verkommen. Die Möglichkeit, etwas zu bestimmen, ihres Schicksals Schmied zu sein, mag für sie eine sinnstiftende Funktion erfüllen, auch wenn es um die Festsetzung des Lebensendes gehen sollte.

Dem zeitlosen Müßiggang von Alice kommt aber seine Quintessenz abhanden, wenn sie die jeweilige Tätigkeit, etwa Fischbrotessen, zum letzten Mal in ihrem Leben macht, fühle sie sich anders, ja besser an. Lässt man Alices Deutung, es handele sich um einen einmaligen und nicht mehr unwiederholbaren Zustand, außen vor, kann man eine Verbesserung ausmachen, die die Ausweglosigkeit der geistigen Lage der jungen Frau in Frage stellt. Ein solches Heilungspotenzial auszuschöpfen, wäre für einen Arzt geradezu eine Pflicht, wobei Gustav zu dieser Einsicht nicht fähig ist, weil seine grundsätzliche Einstellung diese Betrachtungsweise überhaupt nicht zulässt.

Gustavs ärztliches sowie menschliches Anliegen besteht darin, das Sterben zu entdämonisieren, oder, mit seiner späteren Mitarbeiterin Eva gesagt, es „aus den Fesseln der Moral [...] [zu] befreien. ${ }^{\text {"23 }}$ Dabei wird auf ähnliche Denkschemata zurückgegriffen, die T. Macho auf den Punkt bringt :

„In vergangenen Jahrhunderten galt der plötzliche Tod als Unglück, während der allmähliche als guter Tod bewertet wurde, weil es den Menschen erlaubte, ihre irdischen und himmlischen

19 Ebd., S. 28.

20 Ebd., S. 14.

21 Ebd., S. 28.

22 MACHO [Anm. 14], S. 12.

23 BÄRFUSS [Anm. 1], S. 32. 
Angelegenheiten zu ordnen und zu regeln. Heute wird dagegen das allmähliche, verzögerte Sterben mit Hilfe von Patientenverfügungen begrenzt oder gar verhindert, während ein plötzlicher Tod fast schon als Wunschbild des glücklichen Sterbens erscheint." ${ }^{24}$

In der Tat ist Gustavs Tun eine Zusammenführung beider Betrachtungsweisen des Sterbens: Mittels gezielter Ratschläge werden seine Patienten dazu aufgefordert, ihr diesseitiges Dasein so abzuwickeln, dass der Übergang ihrer Habseligkeiten in die Hände der Hinterbliebenen möglichst reibungsfrei vonstattengeht. Mit penibler Achtsamkeit sollen sie eine Angelegenheit nach der anderen klären, damit das Risiko, etwas zu versäumen, klein gehalten wird. Soviel zu den irdischen ,Geschäften', die himmlischen spielen für Gustav und die Mehrheit derjenigen, die sich für den Suizid in seiner Obhut entscheiden, nur eine minderwertige Rolle. Trotzdem bekommt Alice von ihrer Mutter eine Bibel mit auf den Weg gegeben, wohl in der Hoffnung, dass doch noch eine Wende in ihrem Denken eingeleitet werden kann.

\section{Die letzten Augenblicke in Alices Leben}

Nach Ankunft in Gustavs Sterbewohnung klammert sich Alice im Gespräch stur an weltliche Themen, beklagt die miese Lage der Wohnung sowie ihren überhöhten Mietspreis. Ihre Angst vor dem baldigen Tod spiegelt sich auch im Interesse am Speiseangebot einer nahegelegenen Kneipe wider. Dies muss auch Gustav feststellen: „Du bist verkrampft [...] Folge meinem Atem. [...] Du entspannst dich. “25 Es scheint, als könnte die These von T. Macho in diesem Fall bestätigt werden: „Die Zustände des Kranken schwanken zwischen verzweifeltem Todeswunsch und der Angst, jemand werde diesen Wunsch erfüllen: „Nein, ich will nicht sterben!““26 Alices Entschluss zu sterben erfährt offensichtlich Brüche. Jedoch anders als etwa beim krebskranken Engländer John, der Gustavs Sterbeprozedur mehrmals unterbricht und schlussendlich zu Hause stirbt, bringt der Sterbehelfer keinen solchen Respekt auf und fährt in seinem Tun trotz der wachsenden Unsicherheit Alices unbeirrt fort.

Der Grund für Alices Unkonzentriertheit wird gleich in den nächsten Momenten zutage gefördert:

GUSTAV Also. Es geht darum, dass du jetzt das Rohypnol trinkst.

ALICE Habe ich schon genommen.

GUSTAV Nein, Alice, noch nicht.

ALICE Doch, das habe ich schon genommen.

GUSTAV Aber dort steht das Glas noch. Siehst du.

24 MACHO [Anm. 14], S. 420.

25 BÄRFUSS [Anm. 1], S. 49

26 MACHO [Anm. 14], S. 422. 
ALICE Aber ich habe doch vorhin etwas genommen.

GUSTAV Bloß Beruhigungsmittel. ${ }^{27}$

Augenscheinlich aus Furcht vor dem unvorhersehbaren Ausgang der Sterbeprozedur bei einer labilen, gemütskranken Patientin trifft Gustav Maßnahmen, um diesen vorzubeugen. Damit raubt er Alice allerdings die letzten Reste ihrer Willenskraft, macht aus ihrem Tod im Vornherein eine ausgemachte Sache. Damit veruntreut sich Gustav seiner mehrmals lautstark akzentuierten Beteuerung, jeder habe jederzeit die Möglichkeit, seine Absicht aufzugeben und dadurch auch die Absprache mit ihm nichtig zu machen. Dass er Alice ohne ihre ausdrückliche Zustimmung betäubt, um den glimpflichen Ablauf seines Tuns nicht zu gefährden, kann bestenfalls als Heuchelei betrachtet werden. Das Ziel, nämlich die erfolgreiche Tötung eines Menschen, wird nun höher gestellt als die Freiheit, das eigene Leben wieder in Anspruch zu nehmen, wenn auch dem Sterbehelfer einige Unannehmlichkeiten zustoßen können. Bemerkt sei dazu, dass Gustav nicht selbst Hand an seine Patienten anlegt. Sie sind es, die alles in Gang setzen und sich bei dieser konkreten Sterbeweise die Plastiktüte über den Kopf ziehen. Dabei ist vorgesehen, dass der Patient „[a]nders als bei der aktiven Sterbehilfe [...] bis zum Schluss die Kontrolle über sein Handeln [behält]. “28 Diese Bedingung wird bei Alice offensichtlich nicht eingehalten, weil sie nicht im vollen Besitz ihrer geistigen Kräfte ist. ${ }^{29}$

Daher sehe ich die These bestätigt, dass man es hier nicht nur mit Heuchelei zu tun hat, sondern dass ein erfüllter Tatbestand der vorsätzlichen Tötung vorliegt: Spätestens im Moment der geheimen Verabreichung von Betäubungsmedikamenten wird klar, dass Gustav sich sehr wohl bewusst ist, Alice könne ihm gegebenenfalls Probleme bereiten. Er macht sich ihre durch die Geisteskrankheit geminderte Zurechnungsfähigkeit zunutze und greift selber ein, bevor der reibungslose Ablauf in Gefahr gerät. Darüber hinaus muss man ihre beiderseitige Annäherung bedenken, die keineswegs in den Rahmen eines professionellen Arzt-Patient-Verhältnisses passen würde. Trotz anfänglicher Täuschung der Mutter wird tatsächlich eine Art Freundschaft aufgebaut, die für Alice gewiss einer der wichtigsten Beweggründe war, der Mutter den Rücken zu kehren und dem Ruf "going to Switzerland“"30 zu folgen. Aber zurück zur analysierten Sterbeszene. Der letzte mögliche Wendepunkt in Alices Leben wird passiert, als die bereits betäubte und kaum noch rational denkende Patientin ein Glas Rohypnol herunterspült. Statt an dieser Stelle den Todesanlauf zu stoppen und der kriminellen Handlung ein Ende zu bereiten, mahnt Gustav zur Eile. Auf Alices Vorschlag, die verhängnisvolle Plastiktüte selber zu handhaben, geht der Sterbehelfer zwar nicht ein, bleibt dennoch im Raum bis zum allerletzten Moment ihres Lebens, und unterlässt damit eine ärztliche Hilfeleistung. Die bereits erstickende Alice ruft mehrmals nach ihm, um schließlich ihren letzten Ruf an ihre Mutter zu

27 BÄRFUSS [Anm. 1], S. 50.

28 BEDFORD-STROHM, Heinrich: Leben dürfen - Leben müssen. Argumente gegen die Sterbehilfe. S. 41.

29 Vgl. dazu auch HANDBERG, Sven-Erik: Ja zur Sterbehilfe. In: HEDEBY, Berit: Ja zur Sterbehilfe. Mit einem juristischen Kommentar von Rudolf Wassermann. S. 120.

30 Vgl. MACHO [Anm. 14], S. 14; nach https://www.theatlantic.com/health/archive/2014/08/going-to-switzerland-is-a-euphemism-for-assisted-suicide/379182/ 
richten. Über den weiteren Ablauf der Ereignisse bietet der dramatische Text zwar keinerlei Auskunft, es ist aber anzunehmen, dass Gustav der Polizei keinen Bericht darüber erstattet, was an dem inkriminierten Tag vor sich ging. Auf eine Rührung des Gewissens wartet man vergeblich. Die Tatsache, dass Gustav sein Handeln für kernrichtig hält, bestätigt die gleich in den nächsten Szenen vollzogene Sterbebegleitung der Mutter, Lotte. Die ältere Frau, der der Sinn des Lebens, nämlich die Unterstützung und Fürsorge ihrer Tochter genommen wurde, wählt den gleichen Tod wie ihr Kind. Es stellt sich die Frage, ob Gustavs Anweisung, seine Assistentin Eva solle diese Patientin in den Tod begleiten, vielleicht doch noch als eine Art Reue bzw. Feigheit zu deuten wäre. Explizit kommen seine Gefühle, wenn es sie überhaupt gibt, jedoch nicht zum Vorschein.

\section{Mildernde Umstände vorhanden?}

Die Position, in die sich Gustav hineinversetzt sehen will, ist die des einsamen Kämpfers für die Freiheit des Menschen. Mehrmals beteuert er, er sei der einzige, der den Leidenden unter jeden Umständen zur Seite steht, während die anderen ihm ständig mit Angst begegnen und ihn aus ihren Reihen auszuschließen gedenken: „Es ist die Notwendigkeit meiner Arbeit, die sie erschreckt. “31 Obwohl er sich als Geächteter, Ekel und Furcht Hervorrufender, sogar Unberührbarer wahrnimmt, glaubt er, dass es außer ihm niemanden gäbe, der dasselbe tun könnte, wodurch ein Gefühl der uneingeschränkten Unentbehrlichkeit entsteht. Dieses Gefühl könnte insofern drückend ausfallen, dass dadurch eine Wahrnehmung der Sterbehilfe entsteht, die nur in einer Richtung geht und keinen Rücktritt mehr kennt. Der Kulminationspunkt wird bei Alice erreicht, indem mehrere Stärkungsfaktoren ins Spiel gebracht werden: Die persönliche Annäherung von Gustav und Alice führt zur Aufhebung der professionellen Distanz, der Lizenzentzug erhöht Gustavs Bereitschaft nur, sich über den Widerstand der Gesellschaft hinwegzusetzen und diese „ärztliche Tätigkeit“32 den „Unmenschen“33, also den Ärzten zum Trotze zu vollbringen. Der allmählich fortschreitende Distanzverlust gegenüber den Patienten und die Realitätsverweigerung (,[...] ich fühle mich nunmehr getragen vom Einverständnis der Mehrheit $[\ldots]^{“ 34}$ ) lassen Gustav nicht einsehen, in wie weit er von seiner vermeintlich kompromissloslos eingehaltenen, ethisch sauberen Linie abgedriftet ist.

Darf dies als Umstand gewertet werden, der die Instrumentalisierung einer geistig kranken Frau, mit dem Ziel sein eigenes elitäres Einzelgängergefühl zu bestärken, rechtfertigen könnte? Wohl kaum, denn wenn dem so wäre, müsste man mit Gustavs eingeschränkter Mündigkeit argumentieren und somit zugeben, es bestünde kein Unterschied zwischen dem Agens Gustav und dem Patiens Alice.

31 BÄRFUSS [Anm. 1], S. 56.

32 Ebd., S. 41.

33 Ebd., S. 41.

34 Ebd., S. 57. 


\section{Beihilfe zum Tod zwischen Hilfestellung und Willkür}

Als anfangs gesagt wurde, dass die Besetzung der Hauptrolle die Perspektivierung der Sterbehilfe verschärft, wurde die Frage nach einer Trennlinie zwischen Hilfestellung und Urteilen gestellt, welche anhand verkehrter Motivationen gefällt werden. Um diese Aussage noch zu ergänzen, muss hinzugefügt werden, dass auch ,klassische‘ medizinische Fälle für den assistierten Suizid den Eingang ins Stück fanden. Etwa bei Helene Brauchler, einer an Hautkrebs sterbenden Frau, die von ihrer Krankheit schwer verunstaltet, zurückgezogen lebt und deswegen auch ihre Liebsten meidet, fällt es weniger schwer, Gustavs Handeln nachzuvollziehen. Was aber persönlicher Umgang zu sein scheint, entpuppt sich als eine pauschale Herangehensweise zu den sterbewilligen Patienten. Um seinen sich ins pathologische steigernden Wahn, der Einzige zu sein, der den Mut aufbringt, allen ausnahmslos den Tod zu gewährleisten, auch weiterhin speisen zu können, nimmt Gustav die Wünsche seiner Gesamtkundschaft für bare Münze, ohne sie kritisch zu hinterfragen. Dabei kommt es zwangsläufig zu einer Hierarchisierung der eigenen Wünsche und der der Betroffenen, indem die von Gustav im entscheidenden Moment die Oberhand gewinnen und zum Zwangstod von Alice führen.

Alice ist diejenige, die ihren Tod in die Wege leitet - dies steht außer Frage. Derjenige, der den ganzen Vorgang jedoch unentwegt ankurbelt und die Entschlussstärke aufbringt, dem Ganzen ein Ende zu setzen, ist zweifelsohne Gustav. Sein Handeln entbehrt jedoch einer selbstlosen Basis, zieht man seine Besessenheit, für alle ein Heiland zu sein, in Betracht. Der Punkt, wo er die oben besagte Trennlinie überschreitet, stellt Alices Tötung dar, der meines Erachtens durchaus das Prädikat,vorsätzlich‘ anhaftet.

Im Stück Alices Reise in die Schweiz wird ein Szenario skizziert, das beim Umgang mit dem Tod ins Leben gerufen werden kann. Es zeigt, wie persönliche Schwächen und Frustration ein nüchternes Entscheidungsvermögen kontaminiert, wobei sich die Frage stellt, ob solch eine Kompetenz überhaupt von einem Menschen zu erwarten wäre. Die erforderte Neutralität ist allem Anschein nach lediglich ein Konstrukt, dessen Einhaltung einer Einbildung gleich ist. Obwohl auch die am ehesten akzeptablen Seiten von Gustavs Beruf erläutert werden, überschattet der Fall Alice und ihrer Mutter alle vorher ausgetragenen Szenarien. Die menschliche Kompetenz, dieses Thema verantwortungsvoll zu handhaben, wird angezweifelt, womit auch eine klare Meinungsposition bezogen wird: Wenn man sich nämlich daran macht, die Entscheidungshoheit über das Lebensende für sich zu beanspruchen, muss man eines klarstellen: Entweder ist der Mensch imstande, diese Hoheit unter allen Umständen aufrechtzuerhalten, oder es steht ihm nicht zu, sich in den natürlichen Lauf der Dinge einzumischen. Ich wage zu behaupten, dass diese Botschaft in Alices Reise in die Schweiz enthalten ist.

Mgr. et Mg. Jan Trna / 383309@mail.muni.cz

Masarykova univerzita, Filozofická fakulta, Ústav germanistiky, nordistiky a nederlandistiky Arna Nováka 1, 60200 Brno, CZ 